energetic difficulties. More recently, cosmic rays have been shown to be a plausible source, feeding the interstellar medium with light elements that the stars simply inherit from it (along with other elements) when they are born, but there may also be a thermonuclear contribution to ${ }^{7} \mathrm{Li}$.

This little book, based on a series of lectures by a leading member of the Orsay team, gives a clear and concise introduction to the physical and astrophysical considerations involved. The observations are very well summarized and possible explanations put forward, while at the same time making clear what is reasonably well established, what is still somewhat doubtful and what is frankly speculative. The second category contains ideas as to where spallation most probably occurred and the author frankly confesses that he changed his mind on this point during production of the book, although the stellar surface reactions referred to in the title remain relevant as a cause of depletion of light elements if not of their creation.

The book can be warmly recommended as an authoritative and readable introduction to an interesting and rapidly moving field. BERNARD PAGEL

\section{Spectroscopy with Lasers}

Laser Raman Spectroscopy. By Marvin C. Tobin. (Vol. 35.) Pp. $x i+171$. (Wiley Interscience: New York and London, 1971.) $£ 6.50$.

Spectroscopy with Lasers. By W. Demtröder. (Topics in Current Chemistry, Band 17.) Pp. iii +95 . (Springer Verlag: Berlin and New York, 1971.) 28DM ; \$7.70.

LASERS are now relatively familiar tools in many areas of pure and applied science. In spectroscopy they have made possible considerable advances in the practical application of certain wellestablished spectroscopic techniques, particularly those associated with light scattering; they have also led to the discovery of new forms of spectroscopy. Between them these two books treat many of the applications of lasers to spectroscopy.

Using gas lasers for excitation and more refined optical and detection systems, it is now possible to obtain the Raman spectrum of almost any material at least as readily as its infrared spectrum. Since the information derived from Raman spectroscopy is complementary to that obtained from infrared spectroscopy, more and more chemists will now be wanting to use Raman spectroscopy as well as infrared spectroscopy as a routine spectroscopic tool. Professor Tobin's book could prove helpful to such chemists.

Most of the first chapter on the nature of the Raman effect is in fact an account of group theory and normal vibrations; only ten pages are devoted to the theory of the Raman effect which will be unfamiliar to many chemists. A working knowledge of the theory is important particularly when interpreting depolarization ratios and orientation effects in single crystals, and the very condensed account given here is hardly sufficient. A statement on page 12 is misleading. It is stated that for illumination with plane polarized light, $\rho$ may vary from 0 for large $\alpha$ to 0.75 for large $\beta$. In fact $\rho$ is zero if $\beta$ is zero (irrespective of the value of $\alpha$ ) and 0.75 if $\alpha$ is zero (irrespective of the value of $\beta$ ).

The second chapter on experimental methods should prove more useful. Two important topics, the maximization of the scattered light flux reaching the detector from a sample illuminated by a laser source and the factors affecting signal-to-noise ratio, are quite fully treated. Many other practical aspects of Raman spectroscopy are also dealt with, including photomultipliers, methods of signal processing, commercially available Raman spectro. meters, determination of Raman band characteristics (intensities, contours and depolarization ratios), special procedures for the study of single crystals and gases, sample handling and laser sources. Some of these topics are unfortunately given very little space: for example, just one page is devoted to lasers and ten lines to lock-in amplification. The account of sample handling techniques is also far from complete.

The third chapter on interpretation of Raman spectra is mainly devoted to group frequencies. There are useful comparative discussions in which group frequencies in infrared and Raman spectra are compared for many classes of compounds. Unfortunately the information on Raman group frequencies is far less complete that for infrared group frequencies. Indeed Tobin's discussion has had to be based on Raman frequencies obtained with mercury arc excitation and previously listed by Brandmuller and Moser and by Szymanski.

The book ends with an attempt to cover in twelve pages recent applications of Raman spectroscopy to polymers, biological materials, minerals and inorganic glasses, inorganic single crystals, ionic melts and complex ions in solution, water, simple covalent compounds and organic chemistry! In addition to a list of papers specifically referred to in the text, there is a bibliography of publications on Raman spectroscopy for the years 1968-69.

This book is written in a pleasant conversational style which will make it attractive to chemists seeking a practical introduction to the subject.
Professor Demtröder's seventy-nine page essay entitled Spectroscopy with Lasers is a valuable introductory survey of the many and varied applications of lasers in spectroscopy. The topics covered include: absorption and fluorescence spectroscopy; photochemistry; Raman spectroscopy and light scattering; plasma spectroscopy; studies of measurements of lifetimes of excited states and collision processes; microanalysis; saturation spectroscopy and spectroscopy of laser media. There is a useful introductory chapter dealing with the characteristic features of lasers as spectroscopic light sources and a good bibliography of more than 400 references.

Of course, the various topics are not treated in depth but the value of this little book lies in the very wide range of applications which is dealt with. Chemists in particular will find it stimulating to read this essay.

\section{A. LONG}

\section{Crystal Structure}

The Crystalline State. By Peter Gay. Pp. ix +348 . (Oliver and Boyd: Edinburgh, January 1972.) $£$.

THIs book has been designed for undergraduates studying a number of different sciences including crystallography, chemistry, physics, mineralogy and metallurgy. The mathematics required is no more than a first year student may be expected to have. The development of the subject differs from corresponding earlier treatments in that the ideas of symmetry are dealt with in terms of the internal structure of crystals just as much as in terms of their external faces. The study of two-dimensional patterns is followed by that of point groups, morphological problems and space groups. There are three chapters on $X$-ray diffraction from single crystals and from powders. A brief sketch of the physical properties of crystals is followed by a chapter on dislocations. There are four appendices on projections, crystallographic calculations, growth and twinning.

The text is clearly written and bears witness to the long experience of teaching the subject which the author has had. Each chapter is followed by many examples to be worked out and answers are given. The excellence of the text is, unfortunately, marred by the errors in the text-figures: in several, for example, edges of faces which should be parallel to one another are not. Other instances include the failure of a small circle about a point on the primitive circle to cut it at right-angles; incorrect indexing in Fig. $5.5(b)$, giving two positions for the point 001 , and ares which should be circular and are not. Fig. 9.2 has an $\mathrm{S}$-shaped line through Laue spots which 